\title{
Prevalence of Physiological, Pathological and Behavioral Risk Factors in Patients Treated with Antidiabetic Sulfonylureas
}

\author{
Confederat Luminita*, Stefan Roxana, Constantin Sandra, Hăncianu Monica, Profire Lenuta
}

University of Medicine and Pharmacy "Grigore T. Popa", lași , Romania

Diabetes mellitus is an important public health problem because of its increased incidence and its devastating complications. In addition to this, it was observed an increase in prevalence for diabetes risk factors. The aim of this study was to evaluate the frequency of the pathophysiological and behavioral risk factors among patients treated with oral hypoglycemic sulfonylureas. Material and methods: It were analyzed the observation sheets of 200 patients ambulatory treated at the "Providența" Medical Center. Results and discussions: Most of the patients were aged over 65 years, being 48.5\% women and 51.5\% men. The obesity was the most frequent (88\%) risk factor in patients; the majority of them were included in the "overweight" and "first- degree obesity" class (72\%). Regarding associated comorbidities, hypertension was found in $77 \%$ of cases, followed by dyslipidemia. In terms of physiological and behavioral aspects, age and alcohol consumption were predominant. Conclusions: A significant percentage of diabetic patients treated with hypoglycemic sulfonylureas present a high prevalence of the risk factors and even two or three factors associated.

Keywords: diatetic patients, risk factors, pathological, physiological, behavioral

Received: 04 June 2015 / Accepted: 05 August 2015

\section{Background}

Diabetes mellitus (DM) has become one of the most concerning health problem due to its alarming increase in incidence. According to International Diabetes Federation's statistics in 2013 there were 383 million people with diabetes and this number is expected to rise to 582 million in 2035 [1].

In terms of biochemistry and pathophysiology, DM is a chronic metabolic disorder whose main features are high levels of blood glucose and disturbances in carbohydrate, lipid and protein metabolism. This disorder appears to be due to deficiency in insulin secretion by pancreatic $\beta$ cells, associated or not with insulin resistance in peripheral tissues $[2,3]$.

Diabetes is a silent disease and if left untreated or poorly controlled, it can lead to serious macrovascular and microvascular complications. Moreover, according to United Kingdom Prospective Diabetes Study, 40\% of patients diagnosed with type 2 diabetes presented signs or symptoms of diabetic complications $[4,5]$.

Diabetes is nowadays a major concern not only because of its increased incidence and its life-threatening complications, but also because of the alarming increase in the prevalence of risk factors which have a detrimental effect for the evolution of diabetes [6].

The aim of this study was to analyze the presence of physiological, pathological and behavioral risk factors for the development of diabetes and diabetes related complica- tions among patients treated with oral hypoglycemic sulfonylureas.

\section{Material and methods}

A statistical retrospective study on 200 patients ambulatory treated with oral antidiabetic drugs at "Providența" Medical Center, Iași was designed. The patients had in their treatment a sulfonylurea alone or in combination with other oral hypoglycemic agents (biguanides, $\alpha$-glucosidase inhibitors or modulators of incretins).Information as age, sex, area of origin (urban, rural), duration of the disease, associated pathologies and drugs administered simultaneously, diabetes complications, hypoglycemic treatment regimen and side effects experienced were collected from the patients observation sheets. The study was focused on the presence of physiological, pathological and behavioral risk factors for the development of diabetes and diabetes related complications. Data were statistically processed and interpreted using MS Excel software.

\section{Results and discussions}

The patients included in the study were aged between 37 years and 93 years (average age was 67.29 \pm 9.95 years; 67.24 years for women and 67.33 for men). The distribution by age group of patients is shown in table I.

The study group presented a balanced repartition by gender, $48.5 \%$ being women and $51.5 \%$ men (figure 1 ). Patients came from urban areas $(62.5 \%)$ and from rural areas (37.5\%) (figure 2 ). 


\section{Risk factors for diabetes and diabetes related compli- cations}

\section{Obesity/ overweight}

Obesity, measured by body mass index value (BMI) is closely related to the development and evolution to complications of type 2 diabetes, being associated with increased insulin resistance. This is both due to the decrease in the number of insulin receptors and to a post receptor defect where there is a deficiency in tyrosine kinase activity $[3,4,7,8]$.

It was observed, in terms of BMI value, that the majority of studied patients were included in the "overweight" or "first-grade obesity" class (144 patients, 72\%) and only 20 patients $(10 \%)$ had a normal weight. Second-grade or third-grade obesity were found in 29 patients (14.5\%) and 6 patients $(3 \%)$, respectively. The average value of BMI was $30.76 \pm 5.47$ with a minimum of 17.1 and a maximum of 56.8 (Table II).

During the treatment with sulfonylurea agents that were used in combination with other oral hypoglycemic drugs, for 51 patients $(25.5 \%)$ of the 200 patients studied was recorded weight gain. More than a half of them (32 patients) were treated with Glimepiride at moderate to high doses (3-4 mg daily), 18 patients were treated with Gliclazide (60 mg daily) and 1 patient was under treatment with Gliquidone (30 mg daily). It must be noted that 12 of the 51 patients that presented this side effect had in their treatment regimen $\mathrm{B}$ vitamins which are known to promote weight gain. Another aspect which should be mentioned is that 44 of those 51 patients had Metformin in association, which is known to induce weight loss. Considering the complexity of the treatment, it is difficult to establish the contribution of sulfonylureas to weight gain.

\section{Comorbidities associated with diabetes}

Diabetes, hypertension and dyslipidemia are essential components of the metabolic syndrome and are associated with an increased risk of cardiovascular diseases [9]. These diseases are interrelated through their pathophysiologi-

Table I. Distribution by age group

\begin{tabular}{lcc}
\hline Age group & Number of patients & Percentage \\
\hline$<50$ years & 9 & $4.5 \%$ \\
$50-65$ years & 67 & $33.5 \%$ \\
$65-75$ years & 73 & $36.5 \%$ \\
$>75$ years & 51 & $25.5 \%$ \\
\hline
\end{tabular}

Table II. Patients distribution by BIM value

\begin{tabular}{lccc}
\hline BIM value & Class & No. of patients & Percentage \\
\hline$<18.5$ & Underweight & 1 & $0.5 \%$ \\
$18.5-24.9$ & normal weight & 20 & $10 \%$ \\
$25-29.9$ & Overweight & 79 & $39.5 \%$ \\
$30-34.9$ & 1st degree obesity & 65 & $32.5 \%$ \\
$35-35.9$ & 2nd degree obesity & 29 & $14.5 \%$ \\
$>40$ & 3rd degree obesity & 6 & $3 \%$ \\
\hline
\end{tabular}

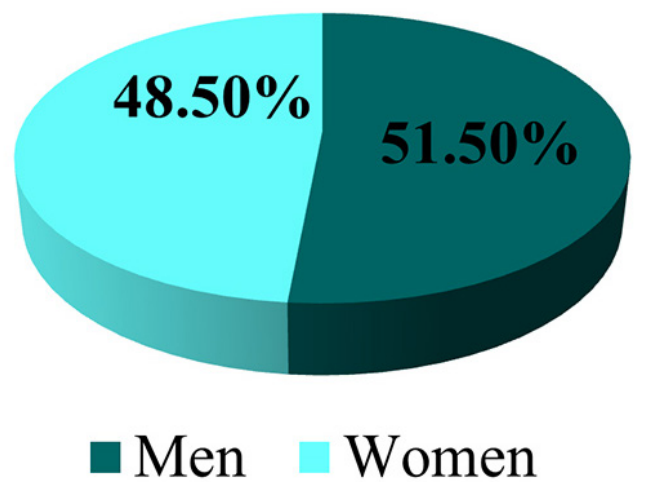

Fig. .1. Gender distribution

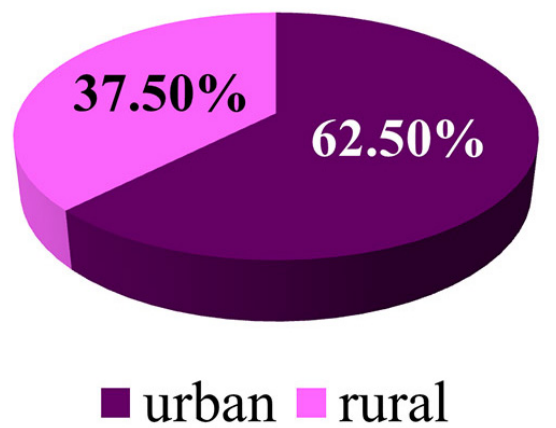

Fig. .2. Environmental distribution

cal mechanisms and a poor control of one can lead to an unfavorable evolution of another. Cardiovascular diseases are the most frequent cause of mortality in patients with diabetes while a good blood pressure and cholesterol control was proved to reduce cardiovascular risk for diabetic patients. On the other hand, a good control of lipid profile is essential for the evolution of diabetes to microvascular complications [10-13].

We could state that hypertension was the second frequent pathological risk factor after obesity in studied patients; 154 (77\% of them) were hypertensive. Dyslipidemia was another risk factor with a significant frequency: hypercholesterolemia was found in 63 patients $(31.5 \%)$, hypertriglyceridemia was observed in 41 patients $(20.5 \%)$ while 53 patients $(26.5 \%$ ) had mixed hyperlipidemia (figure 3). There were only 17 cases in which patients had no comorbidities associated. A total number of 183 patients had one or more diseases considered as risk factors for diabetes and diabetes related complications; the number of patients with more than one comorbidity was quite high (113 patients) (figure 3).

\section{Behavioral and physiological risk factors}

Regarding behavioral risk factors, smoking, alcohol consumption and a sedentary life are considered risk factors for development and evolution of both cardiovascular and metabolic diseases, including diabetes $[10,14]$.

Within the group of studied patients, it was noticed that $14.5 \%$ of patients were chronic alcohol consumers, $8 \%$ were smokers, while $3.5 \%$ had both risk factors. The 


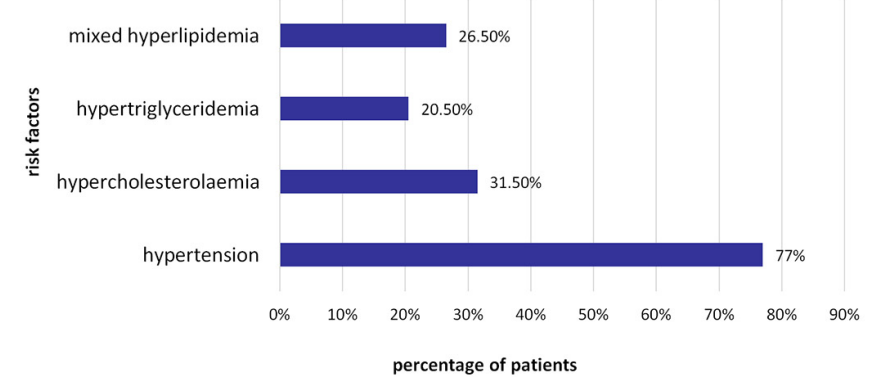

Fig. 3. Associated comorbidities at studied diabetes patients

age over 65 years, considered as physiological risk factor, was found in $56.5 \%$ of cases (figure 4 ).

\section{Conclusions}

We performed a statistical study on 200 diabetic patients treated with oral hypoglycemic sulfonylureas in combination with other drugs, focusing on the analysis of risk factors for diabetes development and diabetes related complications.

The most common risk factor found in our study was obesity of various degrees ( $89.5 \%$ of patients), followed by hypertension $(77 \%)$ and dyslipidemia (31.5\% hypercholesterolemia, $20.5 \%$ hypertriglyceridemia and $26.5 \%$ mixed hyperlipidemia). It was difficult to establish the contribution of sulfonylureas to weight gain because patients that presented it had a poor glycemic control and their treatment included many drugs associated. As for physiological and behavioral risk factors, age over 65 years was found in $56.5 \%$ of cases, followed by alcohol consumption (14.5\%) and smoking (8\%).

A significant part of the patients included in this study presented more than three risk factors associated.

\section{Aknowledgments}

This work was financially supported through the project „Excellence program in multidisciplinary doctoral and postdoctoral research in chronic diseases", contract no. POSDRU/159/1.5/S/133377.

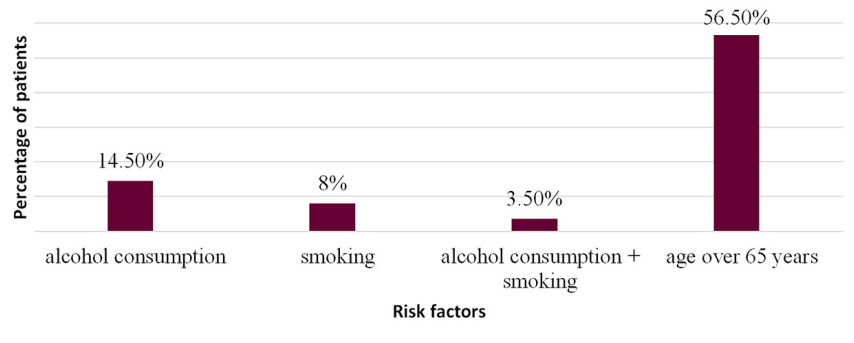

Fig. 4. Behavioral and physiological risk factors identified at studied diabetes patients

\section{References}

1. Guariguata L, Whiting DR, Hambleton I et al. Global estimates of diabetes prevalence for 2013 and projections for 2035. Diab Res Clin Pract. 2014;103(2):137-149.

2. Sonia TA, Sharma CP. Oral Delivery of Insulin. Woodhead Publishing. 2014. 1-57.

3. Virally M, Blickle JF, Girard J, Halimi S, Simon D, Guillausseau PJ. Type 2 diabetes melliuts: epidemiology, pathophysiology, unmet needs and therapeutical perspectives. Diab Metab. 2007;33:231-244.

4. Del Prato S, Pulizzi N. The place of sulfonylureas in the therapy for type 2 diabetes mellitus. Metab Clin Experim. 2006;55(Suppl 1): S20-S27.

5. Germino FW. Noninsulin Treatment of Type 2 Diabetes mellitus in Geriatric Pacients: A review. Clin Therap. 2011; 33(12):1868-1882.

6. Solet JL, Baroux N, Pochet M, et al. Prevalence of type 2 diabetes and other cardiovascular risk factors in Mayotte in 2008: The Maydia study, Diabetes \& Metabolism. 2011;37:201-207.

7. Foster DW. Diabetul zaharat. In: Harrison - Principiile medicinei interne. Bucharest, Romania: Teora, 2003. 2265-2294.

8. Belhatem N, Mohammedi K, Rouzet F, et al. Impact of morbid obesity on the kidney function of patientis with type 2 diabetes. Diabetes Res and Clin Pract. 2015;108(1):143-149.

9. Fukui M, Tanaka M, Toda $\mathrm{H}$, et al. Risk factors for the developement of diabetes mellitus, hypertension and dyslipidemia. Diabetes Res and Clin Pract. 2011,94(1):15-18.

10. Samaranayaka S, Gulliford MC. Trends in cardiovascular risk factors among people with diabetes in a population based study, Health survey for England 1994-2009. Primary Care Diabetes. 2013;7(3):193-198.

11. Taskinen MR, Borén J. New insights into the pathophysiology of dyslipidemia in type 2 diabetes. Atherosclerosis. 2015;239(2): 483-495.

12. Lastra G, Syed S, Kurukulasuriya LR et al., Type 2 Diabetes Mellitus and Hypertension: An Update. Endocrinology and Metabolism Clinics of North America. 2014;43(1):103-122.

13. Frontoni $S$, Solini A, Fioretto $P$, et al. The ideal blood pressure target to prevent cardiovascular disease in type 2 diabetes: A neutral viewpoint. Nutrition, Metabolism and Cardiovascular Diseases. 2014;24(6):577584.

14. Wei X, Meng E, Sufang Yu. A meta-analysis of passive smoking and risk of developing type 2 diabetes mellitus. Diabetes Res and Clin Pract. 2015;107(1):9-14. 